\title{
Paradigm shifts in alternative access for transcatheter aortic valve replacement: An update
}

Lucía Junquera, MD, ${ }^{a}$ Dimitri Kalavrouziotis, MD, ${ }^{\mathrm{b}}$ Eric Dumont, MD, ${ }^{\mathrm{b}}$ Josep Rodés-Cabau, MD, ${ }^{\mathrm{a}}$ and Siamak Mohammadi, MD ${ }^{\mathrm{b}}$

Supplemental material is available online.

Over the last decade, transcatheter aortic valve replacement (TAVR) has evolved from a procedure reserved for high-to-prohibitive surgical risk patients, ${ }^{1-3}$ to an established therapeutic alternative to surgical aortic valve replacement (SAVR) in the low-to-intermediate risk population. ${ }^{4-7}$ The femoral artery is the first-choice approach whenever possible, ${ }^{8}$ as this was the predominant access site used in all of the randomized trials comparing TAVR with SAVR.

Recent registries have shown a significant decrease in the proportion of patients treated through a nontransfemoral (TF) access, from nearly $20 \%$ to $5 \%,{ }^{9-12}$ as a consequence of the iterative technological improvements in transcatheter heart valves (THV) and delivery system profiles. Nonetheless, considering the rapid increase in the number of TAVR procedures performed worldwide, it is reasonable to assume that some proportion of these patients will not be candidates for TF-TAVR. ${ }^{10}$ In these cases, attempting a TF-TAVR despite an unfavorable ileofemoral anatomy may be associated with greater rates of vascular injury and mortality. ${ }^{13,14}$ Furthermore, as TAVR expands toward a lower-risk population, similar outcomes as those achieved by TF access are mandatory to rival the excellent results associated with SAVR in this subset of patients. Several transthoracic (transapical [TA]/transaortic [TAo]) and peripheral

\footnotetext{
From the Departments of ${ }^{\mathrm{a}}$ Cardiology, and ${ }^{\mathrm{b}}$ Cardiac Surgery, Quebec Heart and Lung Institute, Laval University, Quebec City, Quebec, Canada.

Dr Lucía Junquera was supported by a research grant from the Fundación Alfonso Martín Escudero (Madrid, Spain). Dr Josep Rodés-Cabau holds the Research Chair "Fondation Famille Jacques Larivière" for the development of structural heart disease interventions.

Received for publication Jan 27, 2021; revisions received April 11, 2021; accepted for publication April 13, 2021; available ahead of print May 3, 2021.

Address for reprints: Siamak Mohammadi, MD, Department of Cardiac Surgery, Quebec Heart and Lung Institute, Laval University, 2725 chemin Sainte-Foy, Quebec City, Quebec, Canada G1V 4G5 (E-mail: siamak.mohammadi@fmed. ulaval.ca).

J Thorac Cardiovasc Surg 2023;165:1359-70

0022-5223/\$36.00

Copyright (C) 2021 by The American Association for Thoracic Surgery

https://doi.org/10.1016/j.jtcvs.2021.04.075
}

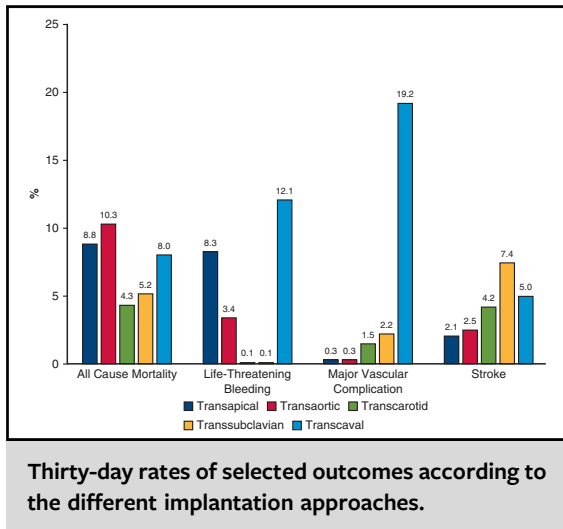

CENTRAL MESSAGE

An in-depth understanding of the risks and benefits of alternatives to transfemoral access is crucial for optimizing the outcomes associated with transcatheter aortic valve replacement. See Commentaries on pages 1371 and 1372 .

(transcarotid [TC], transsubclavian [TS], transcaval $[\mathrm{TCv}])$ vascular access routes were developed as alternatives in the event that TF access was unsuitable. A careful evaluation of patients' comorbidities and vascular anatomy is necessary to choose the best alternative access, as each technique has its advantages and disadvantages (Table 1).

In the early years of TAVR, TF access was associated with improved clinical outcomes compared with other more-invasive vascular approaches. ${ }^{15}$ However, in an intermediate-risk population treated with secondgeneration THVs through a TF approach, the risks of major and/or life-threatening bleeding and major vascular complications were reported to be as high as $12 \%$ and $8 \%$, respectively, raising the question as to whether patients with a nonoptimal $\mathrm{TF}$ access should continue to be treated using this approach. ${ }^{6,7}$ There is increasing evidence suggesting that, to reduce access-related vascular and bleeding complications, as well as the excess early and late mortality associated with these complications, a safe alternative access 
TABLE 1. Relative advantages and disadvantages associated with the different TAVR approaches

\begin{tabular}{|c|c|c|}
\hline & Advantages & Disadvantages \\
\hline Transapical & $\begin{array}{l}\text { - Easy aortic valve crossing } \\
\text { - Excellent THV maneuverability } \\
\text { - Not limited by peripheral vascular anatomy and size }\end{array}$ & $\begin{array}{l}\text { - General anesthesia and mechanical ventilation } \\
\text { - Mini-thoracotomy } \\
\text { - Irreversible damage to apical myocardium possibly limiting } \\
\text { LVEF improvement post-TAVR } \\
\text { - Longer in-hospital stay } \\
\text { - Relative contraindications: } \\
\text { - Chronic respiratory insufficiency } \\
\text { - Severe left ventricular dysfunction } \\
\text { - Left ventricular clot }\end{array}$ \\
\hline Transaortic & $\begin{array}{l}\text { - Excellent THV maneuverability } \\
\text { - No guidewire manipulation of aortic arch and supra-aortic } \\
\text { vessels } \\
\text { - Rapid conversion to full sternotomy if necessary } \\
\text { - Not limited by peripheral vascular anatomy and size }\end{array}$ & $\begin{array}{l}\text { - General anesthesia and mechanical ventilation } \\
\text { - Partial sternotomy (unless suprasternal access) } \\
\text { - Longer in-hospital stay } \\
\text { - Relative contraindications: } \\
\text { - Chronic respiratory insufficiency } \\
\text { - Previous sternotomy } \\
\text { - Porcelain aorta } \\
\text { - Short ascending aorta ( }<6 \mathrm{~cm} \text { from the puncture site to } \\
\text { the aortic annulus) } \\
\text { - Previous chest radiation }\end{array}$ \\
\hline Transcarotid & $\begin{array}{l}\text { - Possibility of local anesthesia and conscious sedation } \\
\text { - Superficial and accessible location of the common carotid } \\
\text { artery } \\
\text { - Common carotid is usually spared of atherosclerotic disease } \\
\text { compared with femoral arteries } \\
\text { - Easy alignment with the aortic annulus } \\
\text { - No guidewire manipulation of aortic arch }\end{array}$ & $\begin{array}{l}\text { - Surgical cut-down is necessary } \\
\text { - Relative contraindications: } \\
\text { - Congenital variants of the aortic arch } \\
\text { - Common carotid artery minimal lumen diameter }<6 \mathrm{~mm} \\
\text { - Contralateral significant }(\geq 50 \%) \text { internal or common } \\
\text { carotid artery stenosis or occlusion }\end{array}$ \\
\hline Transsubclavian & $\begin{array}{l}\text { Possibility of local anesthesia and conscious sedation. } \\
\text { - Proximity to the aortic annulus } \\
\text { - Subclavian artery is usually spared of atherosclerotic } \\
\text { disease compared with femoral arteries }\end{array}$ & $\begin{array}{l}\text { - Alignment with the aortic annulus more difficult } \\
\text { - Deep location, surgical access challenging in patients with } \\
\text { obesity } \\
\text { - Proximity of the brachial plexus } \\
\text { - Bailout technically difficult if vascular complications } \\
\text { - Relative contraindications: } \\
\text { - Subclavian artery minimal lumen diameter }<6 \mathrm{~mm} \\
\text { - Vascular anatomy: tortuosity, stenosis, angulation } \\
\text { - Patent ipsilateral internal mammary artery graft } \\
\text { - Patients with obesity } \\
\text { - Steep subclavian to aortic arch angulation }\end{array}$ \\
\hline Transcaval & $\begin{array}{l}\text { - Fully percutaneous venous access } \\
\text { - Possibility of local anesthesia and conscious } \\
\text { - Sedation }\end{array}$ & $\begin{array}{l}\text { - Extensive preoperative planning from the multislice CT } \\
\text { - Added cost of closure devices } \\
\text { - Presence of intestine within the access pathway } \\
\text { - Relative contraindications: } \\
\text { - Calcification/atheroma/aneurysm/chronic dissection of } \\
\text { descending aorta } \\
\text { - Increased distance between descending aorta and } \\
\text { inferior vena cava }\end{array}$ \\
\hline
\end{tabular}

$\overline{T H V}$, Transcatheter heart valve; $L V E F$, left ventricular ejection fraction; $T A V R$, transcatheter aortic valve replacement; $C T$, computed tomography.

site should be considered based on the individual patient's anatomical characteristics. ${ }^{14}$ This article aims to provide a brief and up-to-date comparative review, focusing on the strengths and weaknesses of the currently available alternative-access TAVR approaches.

\section{TRANSTHORACIC APPROACHES (TA AND TAo) Technical Features}

During the early experience with TAVR procedures, transthoracic approaches were the preferred alternative routes, as the high-profile introducer sheaths precluded 
the use of safe alternative peripheral arterial access. The TA pathway, first performed by Ye and colleagues in $2005,{ }^{16}$ was the first alternative access attempted for TAVR. Despite a need for an anterolateral mini-thoracotomy, general anesthesia, and the insertion of the THV delivery system though the left ventricular apex, TA access was the only anterograde approach that allowed for straightforward guidewire crossing of the aortic valve and excellent THV maneuverability. ${ }^{17}$ Nevertheless, the TA approach has been associated with myocardial damage, and a smaller extent of improvement in left ventricular function after TAVR, ${ }^{18,19}$ as well as various other important procedure-related complications, such as tamponade and, albeit rare, injury to the mitral subvalvular apparatus. Intractable bleeding at the left ventricular apex is also rare but may be a potentially catastrophic complication that often requires cardiopulmonary bypass.

Access involving the ascending aorta was developed as a means of countering the potential complications related to left ventricular apical access in elderly and frail patients, and the TAo route was first described in $2010 .{ }^{20}$ A partial upper sternotomy is performed, extended down to the second or third right intercostal space, although sternalsparing approaches have been described, such as an incision through the suprasternal notch. General anesthesia is required. Previous sternotomy, especially with patent coronary bypass grafts, and a heavily calcified ascending aorta are generally prohibitive.

\section{Outcomes of Transthoracic Access}

Studies reporting the outcomes associated with TA/TAo TAVR are listed in Table 2. Data from large registries of high-risk patients showed 30-day and 1-year mortality rates ranging from $9 \%$ to $14 \%$ and $25 \%$ to $32 \%$ after TATAVR $^{21-25, E 1-E 3}$ and $6 \%$ to $10 \%$ and $19 \%$ to $30 \%$ after TAo-TAVR, ${ }^{24, E 4, E 5}$ respectively. Major vascular complications, major bleeding events, and stroke were similar between TA and TAo approaches. ${ }^{24, \mathrm{E} 2, \mathrm{E} 6}$ Thourani and colleagues $^{24}$ reported a greater rate of 30-day and 1-year unadjusted mortality in the TAo access group, but these differences were no longer statistically significant after a riskadjusted analysis.

\section{Transthoracic Compared With Transfemoral TAVR}

Meaningful comparisons between transthoracic and TF access are problematic, due to the routine use of $\mathrm{TF}$ as the first-line approach, with transthoracic access reserved for cases not amenable to nontransthoracic access, which are generally considered to be at a greater risk. Betweengroup differences could therefore be wrongly attributed to the type of TAVR access, rather than to the patient's preprocedural risk profile, with access usually emerging as an independent predictor of poor outcome. There are a few propensity-score matched studies that suggest that there is no inherent access-related detrimental effect among the transthoracic approaches. ${ }^{\mathrm{E} 7 \mathrm{E} 8}$ However, a majority of comparative studies, even after risk adjustment, report significantly greater rates of major adverse events such as postprocedural major/life-threatening bleeding, acute renal failure, and in-hospital mortality among patients undergoing transthoracic access. ${ }^{15, \text { E9-E13 }}$ Late survival was also reduced when compared with TF-TAVR. ${ }^{23, E 2}$ Stroke risk after the transthoracic approaches is low; the avoidance of retrograde guidewire crossing of the aortic arch, and catheter manipulation in the arch are possible reasons. ${ }^{\text {E14 }}$ Similar rates of neurologic events were reported for TA/ TAo and TF access, both in large multicenter and single-center studies, despite a greater incidence of postprocedural atrial fibrillation in the transthoracic access group. $^{24, \mathrm{E} 8, \mathrm{E} 11, \mathrm{E} 15}$ Functional status and quality of life showed a more modest improvement in TA-TAVR recipients compared with those who underwent TF-TAVR. ${ }^{19, \mathrm{E} 16}$ Finally, the overall costs associated with transthoracic TAVR are greater than TF access, not only as a consequence of longer in-hospital stays but also due to a greater burden of procedure-related complications and re-hospitalizations. ${ }^{\mathrm{E} 10, \mathrm{E} 12}$ It is not surprising, then, to see a progressive decrease in the number of cases performed through a transthoracic approach $(19.2 \%$ in 2010 compared with $3.9 \%$ in 2015 in the FRANCE TAVR registry and $14.5 \%$ in 2012 compared with $6.1 \%$ in 2015 in the Society of Thoracic Surgeons/ American College of Cardiology Transcatheter Valve Therapy Registry [STS/ACC TVT] Registry) in favor of other less-invasive alternative access pathways. ${ }^{11,12}$

\section{PERIPHERAL ARTERIAL APPROACHES (TC AND TS)}

\section{Technical Features-TS Access}

The reduction of THV delivery sheath diameters has progressively encouraged the use of peripheral vessels as the preferred alternative pathway. Transsubclavian or transaxillary access (TS) was developed as one of the first alternative non-TF peripheral arterial approaches. The first case was successfully performed by Ruge and colleagues in 2007. ${ }^{\mathrm{E} 17}$ The subclavian artery can have variable depth from the skin surface depending on body habitus, and the brachial plexus is in very close proximity. There are also concerns about a patent ipsilateral internal mammary artery bypass graft in patients with previous coronary artery bypass grafting. Co-axiality, especially when using the right subclavian approach, may be problematic, especially with an aortoventricular angle $<70^{\circ}$. Subclavian arteries are frequently spared from the atherosclerotic disease process, but wall thickness tends to be thinner due to a less robust media layer when compared with the femoral arteries. ${ }^{\text {E18 }}$ Percutaneous access has been reported, with comparable results regarding procedural and short-term outcomes, 
TABLE 2. Summary of the main transthoracic access studies and studies comparing transthoracic versus transfemoral TAVR

\begin{tabular}{|c|c|c|c|c|c|}
\hline & Authors & Comparative groups & Study type & $\begin{array}{c}\text { Study } \\
\text { period, y }\end{array}$ & Age, $y$ \\
\hline \multirow[t]{4}{*}{$\begin{array}{r}\text { Transthoracic } \\
\text { registries }\end{array}$} & $\begin{array}{c}\text { Wendler et al. SOURCE } \\
\text { REGISTRY }^{\mathrm{E} 3}\end{array}$ & Transapical $(\mathrm{n}=1387)$ & Prospective registry & 2007-2009 & $80.6 \pm 7.1$ \\
\hline & $\begin{array}{l}\text { Bapat et al, Cocchieri et al, } \\
\text { ROUTE REGISTRY E5 }\end{array}$ & Transaortic $(\mathrm{n}=301)$ & Prospective registry & 2013-2015 & $81.7 \pm 5.9$ \\
\hline & $\begin{array}{l}\text { Thourani et al. TVT } \\
\text { REGISTRY }^{24}\end{array}$ & Transapical $(\mathrm{n}=4085)$ & $\begin{array}{l}\text { Prospective registry. } \\
\text { Retrospective analysis }\end{array}$ & 2011-2014 & $82.6 \pm 6.8 \ddagger$ \\
\hline & & Transaortic $(\mathrm{n}=868)$ & & & $83.6 \pm 6.8 \ddagger$ \\
\hline \multirow[t]{7}{*}{$\begin{array}{l}\text { Transfemoral vs } \\
\text { transapical studies }\end{array}$} & $\begin{array}{c}\text { Blackman et al, UK TAVI } \\
\text { REGISTRY }\end{array}$ & Transfemoral $(\mathrm{n}=387)$ & $\begin{array}{l}\text { Prospective registry. } \\
\text { Retrospective analysis }\end{array}$ & 2007-2010 & $82.2 \pm 7.4$ \\
\hline & & Transapical $(\mathrm{n}=408)$ & & & $81.8 \pm 6.9$ \\
\hline & $\begin{array}{c}\text { Schymik et al, SOURCE XT } \\
\text { REGISTRY }^{22}\end{array}$ & Transfemoral $(\mathrm{n}=1685)$ & $\begin{array}{l}\text { Prospective registry. } \\
\text { Retrospective analysis }\end{array}$ & $2010-2011$ & $82.0 \pm 6.5 \ddagger$ \\
\hline & $\begin{array}{c}\text { Blackstone et al PARTNER } \\
1 \mathrm{TA}^{15}\end{array}$ & $\begin{array}{l}\text { Transapical }(\mathrm{n}=894) \\
\text { Transfemoral }(\mathrm{n}=501)\end{array}$ & $\begin{array}{l}\text { Prospective. Propensity score } \\
\text { matched }\end{array}$ & 2007-2012 & $\begin{array}{c}80.1 \pm 6.4 \ddagger \\
85 \pm 6.8\end{array}$ \\
\hline & Doshi et al ${ }^{\mathrm{E} 12}$ & $\begin{array}{c}\text { Transapical }(\mathrm{n}=501) \\
\text { Transfemoral }(\mathrm{n}=3954)\end{array}$ & $\begin{array}{l}\text { Retrospective Propensity score } \\
\text { matched }(3: 1)\end{array}$ & 2012-2014 & $\begin{array}{c}85 \pm 6.3 \\
81.1 \pm 8.5 \ddagger\end{array}$ \\
\hline & Schymik et $\mathrm{al}^{\mathrm{E7}}$ & $\begin{array}{l}\text { Transapical }(\mathrm{n}=1318) \\
\text { Transfemoral }(\mathrm{n}=354)\end{array}$ & $\begin{array}{l}\text { Retrospective Propensity score } \\
\text { matched }\end{array}$ & 2008-2012 & $\begin{array}{c}81.0 \pm 8.1 \ddagger \\
81.7 \pm 5.0\end{array}$ \\
\hline & & Transapical $(\mathrm{n}=354)$ & & & $81.8 \pm 5.9$ \\
\hline \multirow[t]{4}{*}{$\begin{array}{l}\text { Transfemoral vs } \\
\text { transaortic studies }\end{array}$} & $\begin{array}{c}\text { O'Hair et al, CoreValve US High } \\
\text { Risk and Extreme Risk Pivotal } \\
\text { Trials }^{\mathrm{E} 13}\end{array}$ & Transfemoral $(\mathrm{n}=394)$ & $\begin{array}{l}\text { Retrospective Propensity score } \\
\text { matched }\end{array}$ & 2011-2012 & $83.2 \pm 7.6$ \\
\hline & & Transaortic $(\mathrm{n}=394)$ & & & $82.8 \pm 7.6$ \\
\hline & Chollet et $\mathrm{al}^{\mathrm{E} 8}$ & Transfemoral $(\mathrm{n}=124)$ & $\begin{array}{c}\text { Retrospective Propensity score } \\
\text { matched }\end{array}$ & $2012-2015$ & $83.9 \pm 6.2$ \\
\hline & & Transaortic $(\mathrm{n}=124)$ & & & $84.1 \pm 6.4$ \\
\hline
\end{tabular}

Values are \%, unless otherwise indicated. STS PROM, Society of Thoracic Surgeons predicted risk of mortality; Log ES, logistic EuroSCORE; CVA, cerebrovascular accident; $V C$, vascular complication; $L T$, life-threatening; $A K I$, acute kidney injury; $C V$, cardiovascular; $N S$, not significant. *Renal failure requiring dialysis. $\nmid$ Acute kidney injury (stage 2 or 3 ). $\ddagger P<.05$. $§$ Acute kidney injury (stage $1-3$ ). $\|$ Blood loss requiring transfusion.

compared with surgical cut-down. ${ }^{\mathrm{E} 19}$ Percutaneous, and even surgical, access can be challenging, especially in patients with obesity. In addition, the management of vascular complications is not straightforward, as the artery is not in an easily compressible location. The TS pathway is feasible under local anesthesia and conscious sedation, which represents an important advantage over transthoracic approaches. $^{\text {E20 }}$

\section{Technical Features-TC Access}

The TC approach for TAVR was first reported in 2010. E21 $^{\text {. }}$ The approach to the common carotid artery (CCA) is performed by a small surgical cut-down along the medial border of the sternocleidomastoid muscle near the sternal notch and is relatively uncomplicated due to the CCA's superficial location, even in patients with obesity. In addition, the CCAs are often free from atheromatous disease, unlike the internal carotid arteries, and are frequently less diseased in comparison with the femoral arteries, even in patients with multiple cardiovascular risk factors and severe peripheral vascular disease. ${ }^{\mathrm{E} 22} \mathrm{TC}$ access is considered feasible if the CCA minimal lumen diameter is $\geq 6 \mathrm{~mm}$, and theoretically, if there is no contralateral significant $(\geq 70 \%)$ internal or CCA stenosis, or occlusion of the contralateral CCA or internal carotid artery. ${ }^{\mathrm{E} 23, \mathrm{E} 24}$ The left side is usually preferred, as the left CCA offers superior coaxial alignment between the aortic root and the THV delivery sheath, but both sides can be used. The procedure can be done either under general anesthesia or conscious sedation with local anesthesia. ${ }^{\text {E25 }}$ In cases in which a compromise of cerebral perfusion is suspected after placement of the introducer sheath before THV deployment, a temporary femorocarotid small-caliber shunt could be used to ensure adequate cerebral perfusion, although this is rarely necessary. 
TABLE 2. Continued

\begin{tabular}{|c|c|c|c|c|c|c|c|c|c|}
\hline \multicolumn{2}{|c|}{ Risk stratification } & \multicolumn{6}{|c|}{ Periprocedural outcomes } & \multicolumn{2}{|c|}{ Follow-up outcomes } \\
\hline STS PROM & $\log \mathrm{ES}$ & CVA & $\begin{array}{c}\text { Major } \\
\text { VC }\end{array}$ & $\begin{array}{c}\text { Major/LT } \\
\text { bleeding }\end{array}$ & AKI & $\begin{array}{c}\text { In-hospital } \\
\text { stay, d }\end{array}$ & $\begin{array}{c}\text { In-hospital } \\
\text { death }\end{array}$ & $\begin{array}{c}\text { 30-d } \\
\text { mortality/CV } \\
\text { mortality }\end{array}$ & $\begin{array}{c}1-y \\
\text { mortality/CV } \\
\text { mortality }\end{array}$ \\
\hline- & $27.6 \pm 16.1$ & 2.5 & 1.5 & Major: 3.9 & $6.7^{*}$ & - & - & 11.2 & 26.2 \\
\hline $9.0 \pm 7.6$ & - & 1.4 & 3.4 & LT: 3.4 & $9.5 \dagger$ & $9.9 \pm 8.5$ & - & $6.1 / 4.4$ & 19.1 \\
\hline $7.4(5-11)$ & - & 2.1 & 0.3 & - & $38.8 \S$ & $8.5 \pm 6.6_{\ddagger}^{\ddagger}$ & 6.8 & $8.8 \ddagger$ & $25.6 \ddagger$ \\
\hline $8.8(6-13)$ & - & 2.5 & 0.3 & - & $39.6 \ddagger$ & $8.9 \pm 6.4 \ddagger$ & 8.1 & $10.3 \ddagger$ & $30.3 \ddagger$ \\
\hline- & $17.7 \pm 11.1 \ddagger$ & 3.3 & $9.0 \ddagger$ & $18.6 \ddagger \|$ & $3.4 \uparrow *$ & - & - & $4.4 \ddagger$ & $18.1 \ddagger$ \\
\hline - & $22.5 \pm 12.9 \ddagger$ & 3.4 & $2.2 \ddagger$ & $28.7 \neq \|$ & $8.6_{*}^{+*}$ & - & - & $11.2 \ddagger$ & $28.7 \ddagger$ \\
\hline- & $19.8 \pm 11.6 \ddagger$ & 3.4 & 7.9 & $10.9 \ddagger$ & $11.9 \ddagger \S$ & - & 1.5 & $4.2 \ddagger / 1.7 \ddagger$ & $15.0 \ddagger / 6.7 \ddagger$ \\
\hline- & $21.9 \pm 13.7 \ddagger$ & 4.2 & $3.5 \ddagger$ & $20.9 \ddagger$ & $28.1 \ddagger \S$ & - & 2.5 & $10.0 \ddagger / 5.7 \ddagger$ & $27.1 \ddagger / 14.7 \ddagger$ \\
\hline- & - & 2.8 & 6.0 & Major: 4.8 & - & $5(3-8) \ddagger$ & $2.8 \ddagger$ & $3.4 \ddagger$ & - \\
\hline - & - & 3.2 & 3.8 & Major: 7.2 & - & $8(5-15) \ddagger$ & $7.4 \ddagger$ & $9.0 \ddagger$ & - \\
\hline- & - & 2.7 & $4.3 \ddagger$ & $31.1 \neq \|$ & $17.1 \ddagger$ & $7.7 \pm 7.3 \ddagger$ & $4.0_{\ddagger}^{\ddagger}$ & - & - \\
\hline - & - & 2.9 & $1.9 \ddagger$ & $36.4 \ddagger \|$ & $23.9 \ddagger$ & $9.7 \pm 7.8 \ddagger$ & $5.4 \ddagger$ & - & - \\
\hline- & $23.5 \pm 16.3$ & 2.3 & 15.8 & 13.6 & $20.9 \ddagger \S$ & - & - & 8.5 & NS \\
\hline- & $23.0 \pm 15.6$ & 2.0 & $2.5 \ddagger$ & 10.1 & $35.6 \div \S$ & - & - & 5.9 & NS \\
\hline $9.7 \pm 5.0$ & - & $2.6 \ddagger$ & $9.4 \ddagger$ & $35.4 \ddagger$ & $10.0 \ddagger \S$ & $8.4 \pm 8.7 \ddagger$ & - & $4.1 \ddagger / 4.1 \ddagger$ & $23.2 / 17.3 \ddagger$ \\
\hline $9.7 \pm 4.8$ & - & $5.7 \ddagger$ & $4.1 \ddagger$ & $66.7 \ddagger$ & $19.7 \ddagger \S$ & $10.8 \pm 8.2 \ddagger$ & - & $10.9 \ddagger / 10.7 \ddagger$ & $28.1 / 22.2 \ddagger$ \\
\hline $6.5 \pm 3.6$ & - & 4.0 & 2.4 & $2.4 \ddagger$ & $1.7 \dagger$ & $6.4 \pm 1.8 \ddagger$ & 1.6 & 6.5 & 16.1 \\
\hline $6.9 \pm 3.6$ & - & 2.4 & 4.8 & $9.7 \ddagger$ & $3.7 \dagger$ & $9.1 \pm 3.6 \ddagger$ & 5.7 & 7.3 & 15.3 \\
\hline
\end{tabular}

\section{Outcomes of Non-TF Peripheral Arterial Access}

Studies reporting outcomes after TS and TC TAVR are listed in Table 3. In an intermediate-to-high risk population, mortality rates ranging from $3 \%$ to $8 \%$ at 30 days and $13 \%$ to $17 \%$ at 1 year have been reported after TC-TAVR. ${ }^{\text {E25-E29 }}$ Outcomes are very similar for TS-TAVR, with reported 30-day and 1-year mortality rates ranging from 3\% to $10 \%$ and $20 \%$ to $30 \%$, respectively. ${ }^{23, \mathrm{E} 2, \mathrm{E} 9, \mathrm{E} 19, \mathrm{E} 29, \mathrm{E} 30}$ Beurtheret and colleagues ${ }^{\mathrm{E} 31}$ published one of the largest cohorts of TAVR procedures performed though a non-TF peripheral arterial access (TC, $n=911$ vs $\mathrm{TS}, \mathrm{n}=702$ ) from the FRANCE TAVR registry. In secondary analysis, TC-TAVR was associated with a significantly lower rate of vascular complications (TC: $0.2 \%$ vs TS: $1.3 \%$, odds ratio [OR], $0.21 ; 95 \%$ confidence interval [CI], 0.04-0.80, $P=.021$ ), but a greater rate of major bleeding and acute kidney injury, compared with TS-TAVR. ${ }^{\mathrm{E} 31}$ Importantly, stroke rate was similar among TC/TS-TAVR recipients (TC: $3.6 \%$ vs TS: $3.0 \%$, OR, $1.24 ; 95 \% \mathrm{CI}, 0.7-2.2, P=.471) .{ }^{\mathrm{E} 31}$ In another recent study, Kirker and colleagues ${ }^{\mathrm{E} 29}$ published a propensity score-matched comparison of a contemporary cohort of TC $(\mathrm{n}=788)$ and TS $(\mathrm{n}=1576)$ patients who received TAVR from the STS/ACC TVT registry. In this study, in-hospital outcomes were comparable between both peripheral access sites, except for the stroke rate: the TC cohort showed a significantly lower rate of stroke compared with the TS cohort $(4.2 \%$ vs $7.4 \% ; P=.003){ }^{\mathrm{E} 29}$ In addition, patients undergoing TC-TAVR had a shorter procedure and fluoroscopy time, a lower contrast volume, and a shorter intensive care unit/inhospital length of stay ${ }^{\text {E29 }}$ Finally, in another study comparing TC- with TS-TAVR, rates of in-hospital, 30-day, and 1-year mortality were similar between the 2 groups. ${ }^{\mathrm{E} 32}$ 




Values are \%, unless otherwise indicated. STS PROM, Society of Thoracic Surgeons predicted risk of mortality; Log ES, logistic EuroSCORE; CVA, cerebrovascular accident; $V C$, vascular complication; $L T$, life-threatening; $A K I$, acute kidney injury; $C V$, cardiovascular; $N S$, not significant. $* P<.05$. $\dagger$ Acute kidney injury (stage $1-3$ ). $\ddagger$ Renal failure requiring dialysis. $\S$ Acute kidney injury (stage 2 or 3 ). $\|$ Blood loss requiring transfusion. 9 Acute kidney injury (stage 3 ). 
TABLE 3. Continued

\begin{tabular}{|c|c|c|c|c|c|c|c|c|c|c|}
\hline \multirow[b]{2}{*}{ Age, $y$} & \multicolumn{2}{|c|}{ Risk stratification } & \multicolumn{6}{|c|}{ Periprocedural outcomes } & \multicolumn{2}{|c|}{ Follow-up outcomes } \\
\hline & STS PROM & $\log$ ES & CVA & $\begin{array}{c}\text { Major } \\
\text { VC }\end{array}$ & $\begin{array}{c}\text { Major/LT } \\
\text { bleeding }\end{array}$ & AKI & $\begin{array}{c}\text { In-hospital } \\
\text { stay }\end{array}$ & $\begin{array}{c}\text { In-hospital } \\
\text { death }\end{array}$ & $\begin{array}{c}\text { 30-d } \\
\text { mortality/CV } \\
\text { mortality }\end{array}$ & $\begin{array}{c}1-y \\
\text { mortality/CV } \\
\text { mortality }\end{array}$ \\
\hline $81.6 \pm 7.8$ & - & $20.5 \pm 14.3$ & 3.6 & $0.2^{*}$ & Major: $10.0^{*}$ & $4.6 * \dagger$ & - & 3.7 & - & - \\
\hline $82.2 \pm 6.6$ & - & $19.2 \pm 13.5$ & 3.0 & $1.3^{*}$ & Major:6.7* & $2.9 * \dagger$ & - & 4.3 & - & - \\
\hline $82.9 \pm 7.2$ & $15.1 \pm 8.63$ & - & 3.0 & 3.0 & 6.0 & - & - & - & 0 & NS \\
\hline $78.1 \pm 8.2$ & $11.9 \pm 9.5$ & - & 8.0 & 0 & 2.6 & - & - & - & 3.0 & NS \\
\hline $78.9 \pm 8.3^{*}$ & $7.6 \pm 5.5^{*}$ & - & $4.2^{*}$ & 1.5 & LT: 0.1 & $1.5 \ddagger$ & $2(2-4)^{*}$ & 2.5 & $4.3 / 1.2$ & - \\
\hline $78.8 \pm 8.4^{*}$ & $7.6 \pm 5.8^{*}$ & - & $7.4^{*}$ & 2.2 & LT: 0.1 & $0.7 \ddagger$ & $3(2-5)^{*}$ & 3.4 & $5.2 / 1.8$ & - \\
\hline $79.3 \pm 6.7$ & $3.5(2.4-4.9)$ & - & 1.0 & - & 11.5 & $0.0 * \S$ & $7(6-9)^{*}$ & 8.0 & 9.0 & - \\
\hline $78.0 \pm 9.9$ & $3.1(2.2-4.8)$ & - & 4.0 & - & 19.2 & $8.0 * \S$ & $6(4-7)^{*}$ & 5.0 & 5.0 & - \\
\hline $79.7 \pm 8.2$ & $6.2 \pm 5.1$ & - & 2.1 & $3.2^{*}$ & $4.3^{*}$ & $0 * \dagger$ & $6(3-8)^{*}$ & - & 2.1 & - \\
\hline $79.4 \pm 5.4$ & $6.9 \pm 3.5$ & - & 3.5 & $10.7^{*}$ & $19.9 *$ & $12.1 * \dagger$ & $8(6-12)^{*}$ & - & 4.6 & - \\
\hline $78.9 \pm 8.7$ & $7.8 \pm 5.9$ & - & $6.3^{*}$ & 2.5 & LT:0.5 & 0.7 & $3(2-5)$ & - & $5.3^{*}$ & - \\
\hline $79.3 \pm 8.0$ & $8.0 \pm 5.1$ & - & $3.1^{*}$ & 1.7 & LT:0.6 & 2.5 & $6(4-8)$ & - & $8.4^{*}$ & - \\
\hline $79.1 \pm 8.4$ & $7.8 \pm 5.3$ & - & 4.3 & 1.4 & LT: 0.2 & $1.3 \ddagger$ & $3(2-4)^{*}$ & $2.4^{*}$ & $4.2 * / 1.2$ & - \\
\hline $79.2 \pm 8.1$ & $7.8 \pm 5.3$ & - & 3.7 & 1.9 & LT: 0.6 & $2.7 \ddagger$ & $6(4-8)^{*}$ & $5.7^{*}$ & $7.7 * / 2.2$ & - \\
\hline $81.9 \pm 7.3$ & - & $19.4 \pm 13.8$ & 3.4 & $0.7^{*}$ & Major: 8.6 & $3.8 \dagger$ & - & 4.0 & - & - \\
\hline $82.1 \pm 7.6$ & - & $19.9 \pm 13.9$ & 2.2 & $1.4^{*}$ & Major: 7.5 & $2.8 \dagger$ & - & 2.9 & - & - \\
\hline $81.1 \pm 7.6$ & - & $25.9 \pm 6.9^{*}$ & 2.1 & 5.3 & $37.2 * \|$ & $4.2 \ddagger$ & - & - & 3.2 & 31.2 \\
\hline $82.0 \pm 6.5$ & - & $19.5 \pm 4.2^{*}$ & 3.0 & 5.8 & $19.9 * \|$ & $2.7 \ddagger$ & - & - & 5.1 & 21.4 \\
\hline $83(79-87)$ & - & $23.3(14-33)$ & 2.1 & 5.0 & $\begin{array}{c}\text { LT: } 7.8 \\
\text { Major: } 36.2\end{array}$ & $4.3 * \Phi$ & - & 5.0 & - & - \\
\hline $83(79-86)$ & - & $23.7(16-34)$ & 2.1 & 7.8 & $\begin{array}{c}\text { LT: } 5.7 \\
\text { Major: } 30.5\end{array}$ & $9.9 * \Phi$ & - & 4.3 & - & - \\
\hline $80.8 \pm 8.1$ & $9.7 \pm 5.9$ & - & 7.5 & 11.9 & $\begin{array}{c}\text { LT: } 11.4 \\
\text { Major: } 27.8\end{array}$ & 10.0 & - & - & $5.4 / 5.4$ & $23.3 / 19.2$ \\
\hline $80.2 \pm 9.7$ & $9.8 \pm 5.5$ & - & 5.0 & 10.4 & $\begin{array}{c}\text { LT: } 10.4 \\
\text { Major: } 22.8\end{array}$ & 14.4 & - & - & $5.9 / 5.9$ & $24.8 / 20.3$ \\
\hline $81.8(7.7)^{*}$ & - & $20.3 \pm 15.0^{*}$ & $4.4^{*}$ & $3.2^{*}$ & $9.2^{*}$ & 3.3 & - & - & 4.1 & NS \\
\hline $83.2(7.0)^{*}$ & - & $17.3 \pm 11.7^{*}$ & $1.9^{*}$ & $7.7^{*}$ & $4.7^{*}$ & 5.1 & - & - & 3.8 & NS \\
\hline $78(72-82)^{*}$ & $4.7(3.2-6.8)$ & - & 2.4 & 2.4 & 4.7 & 3.2 & $5.7 \pm 3.4$ & 3.2 & $4.8 / 3.9$ & NS \\
\hline $82(76-86)^{*}$ & $4.2(2.8-6.7)$ & - & 3.3 & 4.5 & 6.0 & 6.5 & $5.8 \pm 7.4$ & 2.0 & $2.8 / 2.8$ & NS \\
\hline $81.4 \pm 8.4$ & $6.4 \pm 3.3$ & $24.2 \pm 13.3$ & 2.4 & 1.2 & 0.0 & $0.0 \ddagger$ & $12.4 \pm 7.7$ & - & 8.4 & - \\
\hline $80.0 \pm 7.5$ & $6.7 \pm 4.3$ & $21.3 \pm 12.4$ & 2.8 & 4.9 & 1.2 & $1.1 \ddagger$ & $12.0 \pm 8.7$ & - & 5.0 & - \\
\hline
\end{tabular}




\section{Peripheral Arterial (Non-TF) Access Compared With Transthoracic Access}

Recently published data, including data from our center, showed that, compared with TC/TS access, transthoracic approaches were associated with greater rates of postprocedural bleeding, acute kidney injury, and de novo atrial fibrillation, as well as a longer in-hospital stay and a lower proportion to home discharge. ${ }^{\text {E19,E33-E37 }}$ While some studies found comparable rates of 30-day and 1-year mortality among peripheral non-TF arterial and transthoracic TAVR recipients, ${ }^{\mathrm{E} 33, \mathrm{E} 35, \mathrm{E} 36}$ contemporary data from the STS/ACC TVT registry showed superior survival rates after both TC and TS access ${ }^{\mathrm{E} 19, \mathrm{E} 37, \mathrm{E} 38}$ and a trend toward better 30-day and 2-year survival after TC access. ${ }^{\text {E34 }}$

\section{Peripheral Arterial (Non-TF) Access Compared With Transfemoral Access}

In the study by Beurtheret and colleagues ${ }^{\mathrm{E} 31}$ the main analysis was a propensity score-matched comparison between TF- and TC/TS-TAVR patients $(\mathrm{n}=1613$ in each group). In-hospital outcomes including major bleeding, stroke, acute renal failure, and mortality, were comparable among both groups after matching, despite a greater prognosis-limiting disease burden in the TC/TS group. ${ }^{\mathrm{E} 31}$ Although there was a numerically greater rate of neurologic events in the TC/TS group compared with $\mathrm{TF}$, this was not statistically significant $(3.4 \%$ vs $2.2 \%$; OR, 1.38 ; 95\% CI, 0.88-2.19, $P=.156$ ). Interestingly, the rate of vascular complications was significantly greater in the TF-matched cohort (TC/TS: $0.68 \%$ vs TF: $1.36 \%$; OR, $0.45 ; 95 \%$ CI, $0.21-0.93$, $P=.032) .{ }^{\mathrm{E} 31}$ One hypothesis was that participating centers in that study had a relatively high rate of TFcompared with alternative access-TAVR, implying that many patients with "borderline" ileo-femoral anatomy were undergoing TF-TAVR at the expense of safer alternative access routes.

There are only 3 studies that performed a head-to-head comparison between TC- and TF-TAVR. ${ }^{\text {E23,E28,E39 }}$ Watanabe and colleagues ${ }^{\mathrm{E} 39}$ found no differences in all of the clinical outcomes evaluated in an unadjusted analysis, despite the greater baseline risk profile of TC patients.



FIGURE 1. Thirty-day rates of selected outcomes among patients who underwent a transcatheter aortic valve replacement, according to the different implantation approaches. Thirty-day all-cause mortality, life-threatening bleeding, major vascular complications, and stroke rates from the Society of Thoracic Surgeons/American College of Cardiology Transcatheter Valve Therapy Registry (STS/ACC TVT registry) (except for the transcaval access) are presented. Transapical (Thourani and colleagues, ${ }^{24}$ STS/ACC TVT registry $[n=4085]$ ). Life-threatening bleeding rate from Schymik and colleagues ${ }^{22}$ (SOURCE XT Registry $[\mathrm{n}=894]$ ). Transaortic (Thourani and colleagues, ${ }^{24}$ STS/ACC TVT registry $[\mathrm{n}=868]$ ). Life-threatening bleeding rate from Bapat and colleagues ${ }^{\mathrm{E} 5}$ (ROUTE Registry $[\mathrm{n}=301]$ ). Transcarotid (Kirker and colleagues ${ }^{\mathrm{E} 29}$ STS/ACC TVT registry $[\mathrm{n}=788]$ ). Transsubclavian (Kirker and colleagues ${ }^{\mathrm{E} 29}$ STS/ ACC TVT registry $[n=1576])$ Transcaval (Greenbaum and colleagues ${ }^{\mathrm{E} 40}$ ). 
TAVR PATIENT*

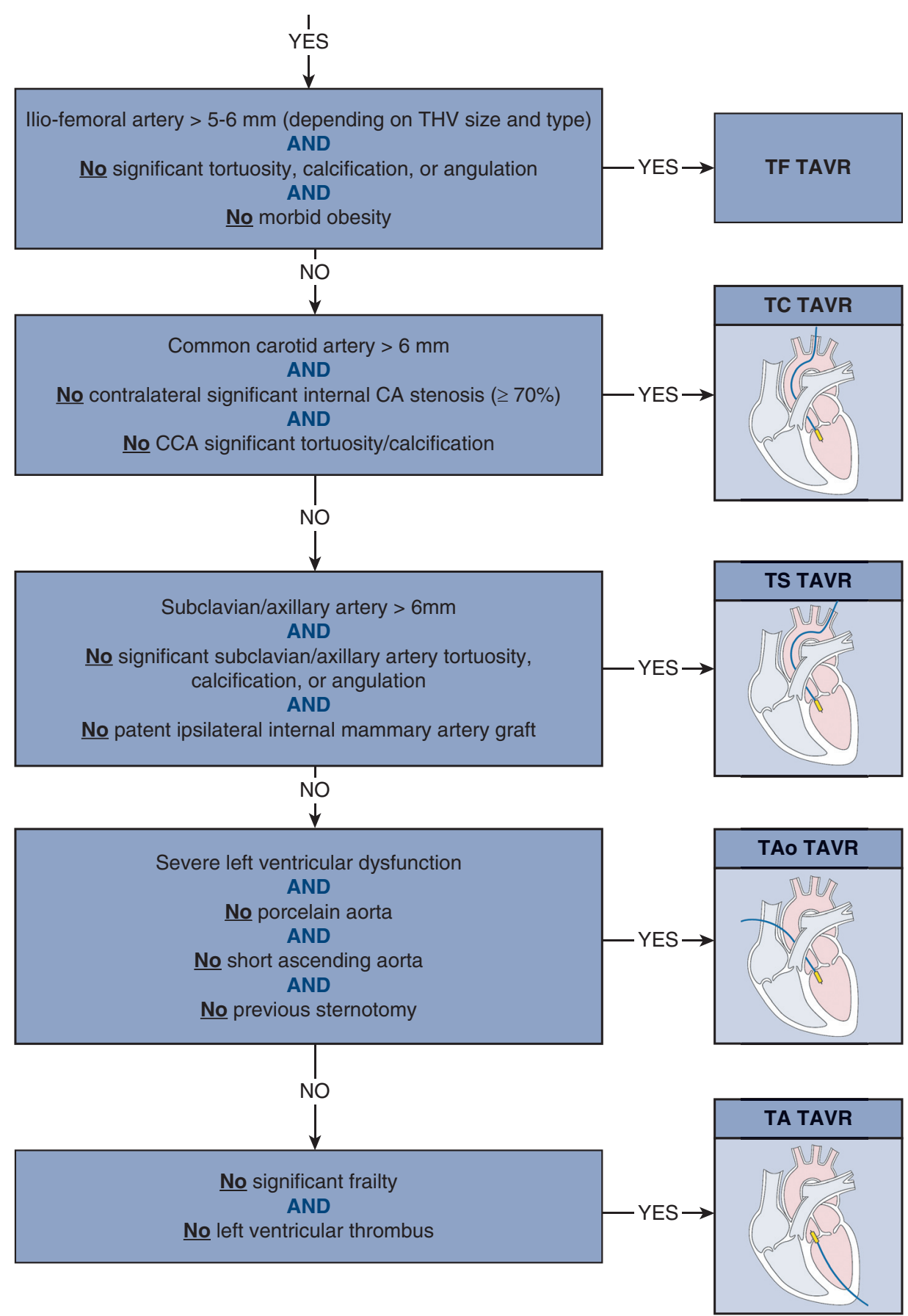

FIGURE 2. Our proposed decision-making algorithm in selecting a nontransfemoral TAVR approach, based on available scientific evidence and the authors' clinical experience, for patients undergoing TAVR. *Patients referred for TAVR are first evaluated by a multidisciplinary heart team, which decides whether TAVR is appropriate. Some patients referred for TAVR are referred for other therapies, such as medical therapy including palliative care, balloon valvuloplasty, or SAVR, but this selection process is beyond the scope of this review. Transcaval and other investigational approaches were not included. $T A V R$, Transcatheter aortic valve replacement; $T H V$, transcatheter heart valve; $T F$, transfemoral; $C A$, carotid artery; $C C A$, common carotid artery; $T C$, transcarotid; TS, transsubclavian; TAo, transaortic; TA, transapical.

Folliguet and colleagues ${ }^{\mathrm{E} 28}$ found no differences in 1- and 2-year mortality rates in a propensity score analysis of $\mathrm{n}=435 \mathrm{TC}$ and $\mathrm{n}=10,598 \mathrm{TF}$ TAVR recipients. However,
TC-TAVR was associated with a greater rate of stroke, bleeding, and renal failure, including the need for dialysis, although a significantly lower rate of vascular 
complications was reported among TC-TAVR patients. ${ }^{\text {E28 }}$ The authors employed inverse probability weighting techniques in their propensity score analysis, but this may not have completely adjusted away the intrinsically higher risk associated with the TC patients. Our own data showed a comparable risk of major vascular complications, acute kidney injury, and major/life-threatening bleeding, among 127 consecutive patients who underwent TC-TAVR compared with 399 patients who underwent TF-TAVR. ${ }^{\text {E23 }}$ Major/life-threatening bleeding related to the TC access site itself occurred in only one patient, and was due to secondary access site bleeding in the remaining patients. ${ }^{\mathrm{E} 23} \mathrm{In}$ addition, the TC-TAVR approach's clinical outcomes in terms of procedural success, early mortality, and stroke rate were comparable with those of TF-TAVR despite a greater baseline risk profile among TC patients. Mortality remains similar out to 1 year of follow-up.

Regarding TS-TAVR, similar rates of 30-day and 1-year mortality were reported when compared to TF-TAV$\mathrm{R}^{\mathrm{E}}{ }^{\mathrm{E}, \mathrm{E} 20, \mathrm{E} 30}$ Bleeding events were greater after TS-TAVR in one study, ${ }^{\mathrm{E} 9}$ but other studies that specifically compared TS and TF access found no differences regarding inhospital outcomes. ${ }^{\mathrm{E} 20, \mathrm{E} 30}$

In summary, encouraging results have been achieved by both TC and TS peripheral arterial access, and concerns about adverse neurologic outcomes, especially after TC access, have largely not been substantiated.

\section{TCv ACCESS}

The $\mathrm{TCv}$ approach is the most recently described pathway for alternative TAVR access. First performed in $2013,{ }^{\mathrm{E} 40}$ this access route shows promise but is generally performed when other options are thought not to be feasible. This approach is possible because the interstitial hydrostatic pressure in the retroperitoneal space exceeds venous pressure, so that blood exiting from the abdominal aorta returns to the venous circulation rather than accumulating in the retroperitoneum. This physiologic mechanism theoretically prevents exsanguination during the time it takes to perform an aorto-caval shunt closure with currently available occluder devices. Persistent aorto-caval fistula is not infrequent after TCv-TAVR, but appears to be well-tolerated. ${ }^{\mathrm{E} 41}$ Computed tomographic evaluation of the descending aorta is needed to determine the optimal caval-aortic crossing trajectory. Initial experience in 100 high-risk patients has shown a high rate of procedural success $(99 \%) .{ }^{\text {E42 }}$ Overall, 30-day major/life-threatening bleeding occurred in $18 \%$ of patients, and major vascular complications in $19 \%$. Thirty-day TCV access-related major/lifethreatening bleeding and major vascular complications occurred in $12 \%$ and $13 \%$ of patients, respectively, typically due to a retroperitoneal hematoma. ${ }^{\mathrm{E} 42}$ The aorto- caval fistula was shown to not be patent immediately after the TAVR procedure in $36 \%$ of the patients, in $66 \%$ of patients at 30 days, and in $86 \%$ at 1 year. ${ }^{E 41}$ Fistula status was not significantly associated with overall survival and subsequent heart failure hospitalizations. ${ }^{\mathrm{E} 41}$ The 30-day and cumulative 1 -year mortality rates were $8 \%$ and $29 \%$, and no patient died or required surgical bailout as a direct consequence of $\mathrm{TCv}$ access. ${ }^{\mathrm{E} 41, \mathrm{E} 42}$ Longer follow-up is mandatory to evaluate long-term tolerance to persistent arteriovenous fistulas, and late events associated with nitinol occluder systems, like late aortic false aneurysm formation, before considering the routine use of $\mathrm{TCv}$ access as a primary non-TF alternative access.

\section{CONCLUSIONS}

Caution must be used when comparing TAVR outcomes between TF and non-TF approaches, as non-TF pathways are always used as a second-option approach in candidates who are not suitable for TF. Therefore, extricating access from baseline risk when examining post-TAVR outcomes remains challenging. Some studies comparing TF with non-TF populations have attempted to risk-adjust using propensity-score matched analyses or other established multivariable techniques, but the lack of randomized clinical trials makes it difficult to reach solid conclusions. Evidence from large nonrandomized, propensity-score matched studies shows similar outcomes between peripheral arterial (TC/TS) and TF TAVR. ${ }^{\mathrm{E} 23, \mathrm{E} 31}$ However, despite its potential advantages and satisfactory outcomes, ${ }^{\mathrm{E} 27, \mathrm{E} 35}$ the TC and TS approaches are available in only $10 \%$ and $39 \%$ of the centers performing TAVR among industrialized nations, respectively, whereas $70 \%$ of those same centers offer TA access as an option. ${ }^{8}$ Recent data from the STS/ACC TVT registry showed that, between 2015 and $2019,41 \%$ of the non-TF cases were performed through a transthoracic approach. ${ }^{\text {E37 }}$ Despite the increased use of peripheral arterial access in more recent years (TA/TAo approaches were surpassed by the TS access in 2017, and by the TC access in 2019), in the first quarter of 2020 transthoracic access was still being employed in $20 \%$ of non-TF cases. ${ }^{\mathrm{E} 29, \mathrm{E} 37}$ The expansion of TAVR to low-risk patients is already under way, and the estimated number of potential TAVR candidates is rapidly increasing. Therefore, there exists a need for a safe alternative approach that can reproduce the results of TF-TAVR. Increasing the penetrance of less-invasive alternative techniques may improve the global results of TAVR by siphoning patients with a high-risk ileofemoral anatomy away from TF-TAVR, and toward nontransthoracic alternative access pathways such as TC or TS access.

The optimal alternative access remains to be elucidated, and direct comparisons between the various non-TF 
strategies must be interpreted with caution, although they may provide us with some guidance as to the relative merits and disadvantages of each approach (Figure 1). Selection of access site should be made on an individual basis for each patient by an experienced multidisciplinary heart team. The heart team may help in identifying those patients with severe iliofemoral disease that increases the risk associated with $\mathrm{TF}$ access and directing those patients to a primary alternative non-TF strategy, thus averting an unplanned femoral arterial repair either by open surgery or stenting post-TAVR. We provide a decision-making algorithm at our institution that takes into consideration each patient's unique baseline clinical and anatomical profile (Figure 2). As experience with alternative peripheral arterial access increases, so may the safety and clinical outcomes associated with alternative-access TAVR prove superior to a "TF for all patients and at any cost" strategy, which is not ideal in an era in which patient safety is the first priority. All TAVR centers must be familiar with at least one alternative approach to ensure optimal TAVR outcomes. Finally, the expertise of each institution's heart team as well as individual center-specific preferences and clinical results are important factors for determining alternative access-site selection. Although randomized studies between TF and nonTF access are unlikely to be performed, further prospective studies comparing a high-risk TF approach versus alternative peripheral access for TAVR are needed, and centers are encouraged to report high-quality registry data, both early and long-term, to continue to improve global TAVR outcomes.

\section{Conflict of Interest Statement}

Dr Josep Rodés-Cabau has received institutional research grants from Medtronic and Edwards Lifesciences. All other authors reported no conflicts of interest.

The Journal policy requires editors and reviewers to disclose conflicts of interest and to decline handling or reviewing manuscripts for which they may have a conflict of interest. The editors and reviewers of this article have no conflicts of interest.

The authors thank Anthoula Kalavrouziotis for providing the illustrations.

\section{References}

1. Leon MB, Smith CR, Mack M, Miller DC, Moses JW, Svensson LG, et al. Transcatheter aortic-valve implantation for aortic stenosis in patients who cannot undergo surgery. N Engl J Med. 2010;363:1597-607.

2. Popma JJ, Adams DH, Reardon MJ, Yakubov SJ, Kleiman NS, Heimansohn D, et al. Transcatheter aortic valve replacement using a self-expanding bioprosthesis in patients with severe aortic stenosis at extreme risk for surgery. J Am Coll Cardiol. 2014;63:1972-81.
3. Smith CR, Leon MB, Mack MJ, Miller DC, Moses JW, Svensson LG, et al. Transcatheter versus surgical aortic-valve replacement in high-risk patients. $N$ Engl J Med. 2011;364:2187-98.

4. Mack MJ, Leon MB, Thourani VH, Makkar R, Kodali SK, Russo M, et al. Transcatheter aortic-valve replacement with a balloon-expandable valve in low-risk patients. N Engl J Med. 2019;380:1695-705

5. Popma JJ, Deeb GM, Yakubov SJ, Mumtaz M, Gada H, O’Hair D, et al. Transcatheter aortic-valve replacement with a self-expanding valve in low-risk patients. N Engl J Med. 2019;380:1706-15.

6. Reardon MJ, Van Mieghem NM, Popma JJ, Kleiman NS, Sondergaard L, Mumtaz M, et al. Surgical or transcatheter aortic-valve replacement in intermediate-risk patients. $N$ Engl J Med. 2017;376:1321-31.

7. Leon MB, Smith CR, Mack MJ, Makkar RR, Svensson LG, Kodali SK, et al. Transcatheter or surgical aortic-valve replacement in intermediate-risk patients. N Engl J Med. 2016;374:1609-20.

8. Cerrato E, Nombela-Franco L, Nazif TM, Eltchaninoff H, Sondergaard L, Ribeiro HB, et al. Evaluation of current practices in transcatheter aortic valve implantation: the WRITTEN (WoRldwIde TAVI ExperieNce) survey. Int J Cardiol. 2017;228:640-7.

9. Siontis GCM, Overtchouk P, Cahill TJ, Modine T, Prendergast B, Praz F, et al. Transcatheter aortic valve implantation vs. surgical aortic valve replacement for treatment of symptomatic severe aortic stenosis: an updated meta-analysis. Eur Heart J. 2019;40:3143-53.

10. Carroll JD, Mack MJ, Vemulapalli S, Herrmann HC, Gleason TG, Hanzel G, et al. STS-ACC TVT registry of transcatheter aortic valve replacement. J Am Coll Cardiol. 2020;76:2492-516.

11. Auffret V, Lefevre T, Van Belle E, Eltchaninoff H, Iung B, Koning R, et al. Temporal trends in transcatheter aortic valve replacement in France: FRANCE 2 to FRANCE TAVI. J Am Coll Cardiol. 2017;70:42-55.

12. Grover FL, Vemulapalli S, Carroll JD, Edwards FH, Mack MJ, Thourani VH, et al. 2016 annual report of the Society of Thoracic Surgeons/American College of Cardiology Transcatheter Valve Therapy Registry. J Am Coll Cardiol. 2017; 69:1215-30

13. Hayashida K, Lefevre T, Chevalier B, Hovasse T, Romano M, Garot P, et al Transfemoral aortic valve implantation new criteria to predict vascular complications. JACC Cardiovasc Interv. 2011;4:851-8.

14. Arnold SV, Manandhar P, Vemulapalli S, Kosinski A, Desai ND, Bavaria JE, et al Impact of short-term complications of TAVR on longer-term outcomes: results from the STS/ACC Transcatheter Valve Therapy Registry. Eur Heart J Qual Care Clin Outcomes. 2021;7:208-13.

15. Blackstone EH, Suri RM, Rajeswaran J, Babaliaros V, Douglas PS, Fearon WF, et al. Propensity-matched comparisons of clinical outcomes after transapical or transfemoral transcatheter aortic valve replacement: a placement of aortic transcatheter valves (PARTNER)-I trial substudy. Circulation. 2015;131: 1989-2000.

16. Ye J, Cheung A, Lichtenstein SV, Carere RG, Thompson CR, Pasupati S, et al. Transapical aortic valve implantation in humans. J Thorac Cardiovasc Surg. 2006;131:1194-6.

17. Nakatsuka D, Tabata M. Transapical approach for transcatheter aortic valve implantation. Ann Cardiothorac Surg. 2017;6:553-4.

18. Rodes-Cabau J, Gutierrez M, Bagur R, De Larochelliere R, Doyle D, Cote M, et al. Incidence, predictive factors, and prognostic value of myocardial injury following uncomplicated transcatheter aortic valve implantation. J Am Coll Cardiol. 2011;57:1988-99.

19. Elmariah S, Fearon WF, Inglessis I, Vlahakes GJ, Lindman BR, Alu MC, et al. Transapical transcatheter aortic valve replacement is associated with increased cardiac mortality in patients with left ventricular dysfunction: insights from the PARTNER I Trial. JACC Cardiovasc Interv. 2017;10:2414-22.

20. Latsios G, Gerckens U, Grube E. Transaortic transcatheter aortic valve implantation: a novel approach for the truly "no-access option" patients. Catheter Cardiovasc Interv. 2010;75:1129-36.

21. Thomas M, Schymik G, Walther T, Himbert D, Lefevre T, Treede H, et al. Thirtyday results of the SAPIEN aortic Bioprosthesis European Outcome (SOURCE) Registry: a European registry of transcatheter aortic valve implantation using the Edwards SAPIEN valve. Circulation. 2010;122:62-9.

22. Schymik G, Lefevre T, Bartorelli AL, Rubino P, Treede H, Walther T, et al. European experience with the second-generation Edwards SAPIEN XT 
transcatheter heart valve in patients with severe aortic stenosis: 1-year outcomes from the SOURCE XT Registry. JACC Cardiovasc Interv. 2015;8:657-69.

23. Gilard M, Eltchaninoff H, Iung B, Donzeau-Gouge P, Chevreul K, Fajadet J, et al. Registry of transcatheter aortic-valve implantation in high-risk patients. $N$ Engl J Med. 2012;366:1705-15.

24. Thourani VH, Jensen HA, Babaliaros V, Suri R, Vemulapalli S, Dai D, et al. Transapical and transaortic transcatheter aortic valve replacement in the United States. Ann Thorac Surg. 2015;100:1718-26; discussion 1726-7.
25. Mack MJ, Brennan JM, Brindis R, Carroll J, Edwards F, Grover F, et al. Outcomes following transcatheter aortic valve replacement in the United States. JAMA. 2013;310:2069-77.

Key Words: aortic stenosis, transcatheter aortic valve replacement, alternative access, transcarotid, transsubclavian, transaortic, transapical 


\section{E-References}

E1. Di Mario C, Eltchaninoff H, Moat N, Goicolea J, Ussia GP, Kala P, et al. The 2011-12 pilot European Sentinel Registry of Transcatheter Aortic Valve Implantation: in-hospital results in 4,571 patients. EuroIntervention. 2013;8: 1362-71.

E2. Frohlich GM, Baxter PD, Malkin CJ, Scott DJ, Moat NE, Hildick-Smith D, et al. Comparative survival after transapical, direct aortic, and subclavian transcatheter aortic valve implantation (data from the UK TAVI registry). Am J Cardiol. 2015;116:1555-9.

E3. Wendler O, Walther T, Schroefel H, Lange R, Treede H, Fusari M, et al. Transapical aortic valve implantation: mid-term outcome from the SOURCE registry. Eur J Cardiothorac Surg. 2013;43:505-11; discussion 511-2.

E4. Cocchieri R, Petzina R, Romano M, Jagielak D, Bonaros N, Aiello M, et al. Outcomes after transaortic transcatheter aortic valve implantation: long-term findings from the European ROUTE†. Eur J Cardiothorac Surg. 2019;55: 737-43.

E5. Bapat V, Frank D, Cocchieri R, Jagielak D, Bonaros N, Aiello M, et al. Transcatheter aortic valve replacement using transaortic access: experience from the multicenter, multinational, prospective ROUTE Registry. JACC Cardiovasc Interv. 2016:9:1815-22.

E6. KE OS, Hurley ET, Segurado R, Sugrue D, Hurley JP. Transaortic TAVI is a valid alternative to transapical approach. J Card Surg. 2015;30:381-90.

E7. Schymik G, Wurth A, Bramlage P, Herbinger T, Heimeshoff M, Pilz L, et al. Long-term results of transapical versus transfemoral TAVI in a real world population of 1000 patients with severe symptomatic aortic stenosis. Circ Cardiovasc Interv. 2015;8:e00761.

E8. Chollet T, Marcheix B, Boudou N, Elbaz M, Campelo-Parada F, Bataille V, et al. Propensity-matched comparison of clinical outcomes after transaortic versus transfemoral aortic valve replacement. EuroIntervention. 2018;14: $750-7$.

E9. Blackman DJ, Baxter PD, Gale CP, Moat NE, Maccarthy PA, Hildick-Smith D, et al. Do outcomes from transcatheter aortic valve implantation vary according to access route and valve type? The UK TAVI Registry. J Interv Cardiol. 2014; 27:86-95.

E10. McCarthy FH, Spragan DD, Savino D, Dibble T, Hoedt AC, McDermott KM, et al. Outcomes, readmissions, and costs in transfemoral and alterative access transcatheter aortic valve replacement in the US Medicare population. J Thorac Cardiovasc Surg. 2017;154:1224-32.e1.

E11. Arai T, Romano M, Lefevre T, Hovasse T, Farge A, Le Houerou D, et al. Direct comparison of feasibility and safety of transfemoral versus transaortic versus transapical transcatheter aortic valve replacement. JACC Cardiovasc Interv. 2016;9:2320-5.

E12. Doshi R, Shah P, Meraj PM. In-hospital outcomes comparison of transfemoral vs transapical transcatheter aortic valve replacement in propensity-matched cohorts with severe aortic stenosis. Clin Cardiol. 2018;41:326-32.

E13. O'Hair DP, Bajwa TK, Popma JJ, Watson DR, Yakubov SJ, Adams DH, et al. Direct aortic access for transcatheter aortic valve replacement using a selfexpanding device. Ann Thorac Surg. 2018;105:484-90.

E14. Eggebrecht H, Schmermund A, Voigtlander T, Kahlert P, Erbel R, Mehta RH. Risk of stroke after transcatheter aortic valve implantation (TAVI): a metaanalysis of 10,037 published patients. EuroIntervention. 2012;8:129-38.

E15. Athappan G, Gajulapalli RD, Sengodan P, Bhardwaj A, Ellis SG, Svensson L, et al. Influence of transcatheter aortic valve replacement strategy and valve design on stroke after transcatheter aortic valve replacement: a meta-analysis and systematic review of literature. J Am Coll Cardiol. 2014;63:2101-10.

E16. Lange R, Beckmann A, Neumann T, Krane M, Deutsch MA, Landwehr S, et al. Quality of life after transcatheter aortic valve replacement: prospective data from GARY (German Aortic Valve Registry). JACC Cardiovasc Interv. 2016; 9:2541-54.

E17. Ruge H, Lange R, Bleiziffer S, Hutter A, Mazzitelli D, Will A, et al. First successful aortic valve implantation with the CoreValve ReValving System via right subclavian artery access: a case report. Heart Surg Forum. 2008;11: E323-4.

E18. Arnett DM, Lee JC, Harms MA, Kearney KE, Ramos M, Smith BM, et al. Caliber and fitness of the axillary artery as a conduit for large-bore cardiovascular procedures. Catheter Cardiovasc Interv. 2018;91:150-6.

E19. Dahle TG, Kaneko T, McCabe JM. Outcomes following subclavian and axillary artery access for transcatheter aortic valve replacement: Society of the Thoracic Surgeons/American College of Cardiology TVT Registry Report. JACC Cardiovasc Interv. 2019;12:662-9.
E20. Petronio AS, De Carlo M, Bedogni F, Maisano F, Ettori F, Klugmann S, et al 2-year results of CoreValve implantation through the subclavian access: a propensity-matched comparison with the femoral access. J Am Coll Cardiol. 2012;60:502-7.

E21. Modine T, Lemesle G, Azzaoui R, Sudre A. Aortic valve implantation with the CoreValve ReValving System via left carotid artery access: first case report. $J$ Thorac Cardiovasc Surg. 2010;140:928-9.

E22. Laclaustra M, Casasnovas JA, Fernandez-Ortiz A, Fuster V, Leon-Latre M, Jimenez-Borreguero LJ, et al. Femoral and carotid subclinical atherosclerosis association with risk factors and coronary calcium: the AWHS Study. J Am Coll Cardiol. 2016;67:1263-74.

E23. Junquera L, Kalavrouziotis D, Côté M, Dumont E, Paradis JM DeLarochellière R, et al. Results of transcarotid compared with transfemoral transcatheter aortic valve replacement. J Thorac Cardiovasc Surg. April 13, 2020 [Epub ahead of print].

E24. Folliguet T, Laurent N, Bertram M, Zannis K, Elfarra M, Vanhuyse F, et al Transcarotid transcatheter aortic valve implantation: multicentre experience in France. Eur J Cardiothorac Surg. 2018;53:157-61.

E25. Debry N, Delhaye C, Azmoun A, Ramadan R, Fradi S, Brenot P, et al. Transcarotid transcatheter aortic valve replacement: general or local anesthesia. JACC Cardiovasc Interv. 2016;9:2113-20.

E26. Overtchouk P, Folliguet T, Pinaud F, Fouquet O, Pernot M, Bonnet G, et al Transcarotid approach for transcatheter aortic valve replacement with the $\mathrm{Sa}$ pien 3 prosthesis: a Multicenter French Registry. JACC Cardiovasc Interv. 2019;12:413-9.

E27. Mylotte D, Sudre A, Teiger E, Obadia JF, Lee M, Spence M, et al. Transcarotic transcatheter aortic valve replacement: feasibility and safety. JACC CardiovasC Interv. 2016;9:472-80.

E28. Folliguet TA, Teiger E, Beurtheret S, Modine T, Lefevre T, Van Belle E, et al Carotid versus femoral access for transcatheter aortic valve implantation: a propensity score inverse probability weighting study. Eur J Cardiothorac Surg. 2019;56:1140-6.

E29. Kirker E, Korngold E, Hodson RW, Jones BM, McKay R, Cheema M, et al Transcarotid versus subclavian/axillary access for transcatheter aortic valve replacement with SAPIEN 3. Ann Thorac Surg. 2020;110:1892-7.

E30. Gleason TG, Schindler JT, Hagberg RC, Deeb GM, Adams DH, Conte JV, et al Subclavian/axillary access for self-expanding transcatheter aortic valve replacement renders equivalent outcomes as transfemoral. Ann Thorac Surg. 2018; $105: 477-83$.

E31. Beurtheret S, Karam N, Resseguier N, Houel R, Modine T, Folliguet T, et al Femoral versus nonfemoral peripheral access for transcatheter aortic valve replacement. J Am Coll Cardiol. 2019;74:2728-39.

E32. Amer MR, Mosleh W, Joshi S, Mather JF, El-Mallah W, Cheema M, et al Comparative outcomes of transcarotid and transsubclavian transcatheter aortic valve replacement. Ann Thorac Surg. 2020;109:49-56.

E33. Beve M, Auffret V, Belhaj Soulami R, Tomasi J, Anselmi A, Roisne A, et al Comparison of the transarterial and transthoracic approaches in nontransfemoral transcatheter aortic valve implantation. Am J Cardiol. 2019;123: 1501-9.

E34. Allen K, Chhatriwalla A, Cohen D, Saxon J, Hawa Z, Kennedy K, et al. Transcarotid versus transapical and transaortic access for transcatheter aortic valve replacement. Ann Thorac Surg. 2019;108:715-22.

E35. Chamandi C, Abi-Akar R, Rodes-Cabau J, Blanchard D, Dumont E, Spaulding C, et al. Transcarotid compared with other alternative access routes for transcatheter aortic valve replacement. Circ Cardiovasc Interv. 2018;11 e006388.

E36. Ciuca C, Tarantini G, Latib A, Gasparetto V, Savini C, Di Eusanio M, et al. Trans-subclavian versus transapical access for transcatheter aortic valve implantation: a multicenter study. Catheter Cardiovasc Interv. 2016;87:332-8.

E37. Allen KB, Chhatriwalla AK, Saxon J, Hermiller J, Heimansohn D, Moainie S, et al. Transcarotid versus transthoracic access for transcatheter aortic valve replacement: a propensity-matched analysis. J Thorac Cardiovasc Surg. October 21, 2020 [Epub ahead of print].

E38. Allen KB, Chhatriwalla AK, Saxon J, Hermiller J, Heimansohn D, Moainie S, et al. Reply: transcarotid trumps transapical/direct aortic access for transcatheter aortic valve replacement-it's a no brainer! J Thorac Cardiovasc Surg. February 22, 2021 [Epub ahead of print].

E39. Watanabe M, Takahashi S, Yamaoka H, Sueda T, Piperata A, Zirphile X, et al Comparison of transcarotid vs. transfemoral transcatheter aortic valve implantation. Circ J. 2018;82:2518-22. 
E40. Greenbaum AB, O’Neill WW, Paone G, Guerrero ME, Wyman JF, Cooper RL, et al. Caval-aortic access to allow transcatheter aortic valve replacement in otherwise ineligible patients: initial human experience. J Am Coll Cardiol. 2014:63:2795-804.

E41. Lederman RJ, Babaliaros VC, Rogers T, Stine AM, Chen MY, Muhammad KI, et al. The fate of transcaval access tracts: 12-month results of the prospective
NHLBI transcaval transcatheter aortic valve replacement study. JACC Cardiovasc Interv. 2019;12:448-56.

E42. Greenbaum AB, Babaliaros VC, Chen MY, Stine AM, Rogers T, O'Neill WW, et al. Transcaval access and closure for transcatheter aortic valve replacement: a prospective investigation. J Am Coll Cardiol. 2017; 69:511-21. 